\title{
Fast Rotating Stars: Effect of the Aspect Angle on Line Profiles
}

\author{
Y. Frémat ${ }^{1}$, J. Zorec ${ }^{2}$, A.M. Hubert ${ }^{1}$, M. Floquet ${ }^{1}$, N. Leister ${ }^{3}$, R. \\ Levenhagen $^{3}$, J. Chauville ${ }^{1}$, and D. Ballereau ${ }^{1}$ \\ 1 Observatoire de Paris, Section d'Astrophysique de Meudon, GEPI, \\ FRE K 2459, 5 Place Jules Janssen, 92195 Meudon CEDEX, France \\ ${ }^{2}$ Institut d'Astrophysique de Paris, CNRS, 98bis Boulevard Arago, \\ 75014 Paris, France \\ ${ }^{3}$ Instituto de Astronomia, Geofisica e ciencias Atmosféricas, Brazil
}

\begin{abstract}
Fast rotation is expected to flatten the star and to produce non uniform temperature and density distributions (i.e. gravitational darkening). While the flattening mostly increases the absolute flux level of the energy distribution, gravitational darkening makes an equator-on star apparently cooler than a star seen through the pole. Both effects (Collins et al. 1991) influence the colours and the location of the star in the HR diagram but also, in a more subtle way, its spectral line profiles. More particularly, in early B type stars, gravitational darkening tends to privilege at the poles the formation of the ions with the highest ionization potentials and directly affects line formation. Consequently, most spectral line shapes - and especially the weakest ones - become aspect angle dependent which in several cases may play a role in the fundamental parameter determination procedures or even in the determination of stellar chemical abundances.
\end{abstract}

\section{Introduction}

Recent studies showed that fast rotation influences the evolution and the atmospheric chemical composition of massive stars (Meynet \& Maeder 2000). But it has also been known for a long time that it provokes a stellar flattening and a gravitational darkening which affects in several ways the shape of spectral lines and continua (Collins et al. 1991). In the present work, we study the amplitude of these effects on the determination of the fundamental parameters (effective temperature, superficial gravity, projected rotation velocity ...) and on the computation of stellar abundances.

\section{Adopted Methodology}

To understand the effects of fast rotation on stellar spectra, we built up a computer code (FASTROT) using a procedure described by Collins et al. (1991, see references therein) but slightly modified to account better for the polar flattening. In these computations, we assume solid rotation and use the von Zeipel theorem to characterize the effects of gravitational darkening on temperature. 
The stellar photosphere is then replaced by a mesh of plane parallel model atmospheres that each describe the local temperature and density distributions.

The local model atmospheres are taken to be those of Kurucz (1994) obtained in LTE with the ATLAS9 code. The energy level populations of the atoms and ions of interest are then computed in NLTE by resolving the statistical equilibrium using the TLUSTY program developed by Hubeny and Lanz (1995, see references therein). Atomic models are built up with the MODION IDL package using data available in the TOPBASE database.

\section{Discussion}

Due to the non uniform temperature distribution, those transitions belonging to ions with the highest ionization potentials will be favored in the polar regions while the others will be mostly formed at the equator. This selective line formation affects the determination of photospheric abundances and generally leads to the underestimate of $v \sin i$. The study of fast rotating early B type stars shows that:

- Species with low ionization potential should be preferred to determine more accurately $v \sin i$.

- The effects of stellar flattening on the absolute flux level are the strongest in pole-on stars while gravitational darkening effects on the line profiles are the strongest if they are seen by the equator.

- Gravitational darkening generally leads to an overestimate or an underestimate of the carbon, nitrogen and oxygen abundances in their pole-on or equator-on configuration respectively. In the worst cases these effects may exceed 0.1 dex.

- The determination of fundamental parameters in fast rotating equatoron stars without accounting for gravitational darkening produces effective temperatures and superficial gravities lower than when it is taken into account.

Acknowledgments. This work has been supported by a Marie Curie Individual Fellowship under contract number HPMF-CT-2000-00497.

\section{References}

Collins, G. W., Truax, R. J., \& Cranmer, S. R. 1991, ApJS 77, 541

Hubeny, I. \& Lanz, T. 1995, ApJ 439, 875

Kurucz, R. 1994, CD-ROM No. 19.

Meynet, G. \& Maeder, A. 2000, A\&A 361, 101 